\title{
Encapsulation of Astaxanthin-enriched Camelina oil Extract in Ovalbumin/gum Arabic Stabilized Emulsion With/without Crosslinking by Tannic Acid
}

\author{
Liyang Xie, ${ }^{1}$ Ozan $\mathrm{Ciftci}^{1}$ and Yue Zhang ${ }^{1,2, *}$
}

\begin{abstract}
Astaxanthin, as a high-value red carotenoid, has attracted much attention in recent years. The astaxanthin-enriched camelina oil extract can be a promising natural antioxidant and colorant. Therefore, effort is demanded to overcome the low oxidative stability and low water-solubility of the oil extract to broaden application fields. In this study, egg albumin (EA) and gum arabic (GA) were used as a combination to emulsify camelina oil extract. The resultant emulsions were further crosslinked by tannic acid, a natural phenolic cross-linker. The entrapment efficiency of astaxanthin in EA/GA stabilized emulsions was around $70 \%$. The size of emulsions at different $\mathrm{pH}$ conditions was investigated, and the smallest diameter was observed at $\mathrm{pH}$ 7. But the best astaxanthin retention was achieved at $\mathrm{pH} 5$ during storage at room temperature. After crosslinking by tannic acid, emulsions showed better protection of astaxanthin against UV light and heat. Our findings provided a promising emulsion system to encapsulate astaxanthin-enriched camelina oil.
\end{abstract}

Keywords: Astaxanthin; Tannic acid; Emulsion; Crosslinking; Encapsulation

Received: 3 August 2020; Accepted: 3 September 2020.

Article type: Research article.

\section{Introduction}

Astaxanthin, a lipophilic bioactive compound, belongs to the carotenoid family which normally found in marine animals such as salmon, shrimp, and lobster. ${ }^{[1]}$ Due to the presence of hydroxyl and ketonic functional groups in its structure, astaxanthin has been reported to exhibit relatively higher antioxidant activity than vitamin $\mathrm{E}$ and beta-carotene. ${ }^{[1,2]}$ Several healthy benefits of astaxanthin have also been reported, including protection against inflammation, cancer, and inhibition of oxidative stress and cardiovascular disease. ${ }^{[3,4]}$ In aquaculture, astaxanthin is used as a natural colorant, particularly for salmonids. ${ }^{[2]}$ However, current sources of natural astaxanthin are limited and fall short of global demand. In our previous study, ${ }^{[5]}$ astaxanthin was enriched in engineered camelina seed, an underutilized oilseed in the USA with high oil content especially the omega-3 fatty

\footnotetext{
1 Department of Food Science and Technology, University of Nebraska-Lincoln, Lincoln, NE, 68588, United States

${ }^{2}$ School of Food Science and Biotechnology, Zhejiang Gongshang University, Hangzhou 310018, China

*E-mail: zhangyue@zjgsu.edu.cn or yue.zhang@unl.edu (Y. Zhang)
}

acids. ${ }^{[6]}$ The astaxanthin-enriched camelina seed oil could be a potential resource of natural antioxidant and colorant. Astaxanthin is highly unsaturated and likely to degrade due to the presence of light, oxygen and heat stress. Similar to camelina oil, which also has low oxidative stability due to the high omega-3 fatty acid content. Besides, the low solubility of astaxanthin in water limits its food applications. Therefore, encapsulation strategies including emulsification, incorporation into liposomes and solid lipid nanoparticles are frequently used to protect astaxanthin against degradation. ${ }^{[7-9]}$

Emulsification is one of the most common methods to encapsulate oily material. However, due to several physicochemical mechanisms, such as gravitational separation, flocculation, and Ostwald ripening, emulsion systems have a propensity to break down. ${ }^{[10]}$ In order to stabilize the emulsion, emulsifiers are normally utilized, including both synthetic (e.g. Tween 80) and natural emulsifiers (e.g. proteins, polysaccharides, and phospholipids). Due to the safety concerns about those synthetic emulsifiers, there is a growing interest in utilizing natural emulsifiers in the food industry. Chicken egg albumin (EA), a common commercial albumin product, is the main constituent of egg white protein $(\sim 65 \%)$. It is an important food ingredient and frequently used as foam and emulsion 
stabilizing agent. ${ }^{[11]}$ Gum arabic (GA), an amphiphilic polysaccharide, is widely used in food industry as stabilizer, thickening agent, and emulsifier. ${ }^{[12]}$ GA has high water solubility, low viscosity, and shows persistent stability in a wide $\mathrm{pH}$ range, making it ideal as biopolymer emulsifier for flavor/beverage emulsions. A combination of EA/GA has also been used to stabilize emulsion, and the different preparation methods may result in different emulsion structures. ${ }^{[13]}$ In this study, the oil droplet was coated by a single layer composed of EA/GA complexes. But the dissociation between EA and GA may still occur due to environmental stresses resulting in an unstable emulsion.

Crosslinking techniques (e.g. chemical, physical, and enzymatic) of biopolymers are effective to enhance emulsion stability and functionality. ${ }^{[14]}$ Tannic acid, belonging to polyphenol family, is abundant with hydroxyl groups. It has been shown that tannic acid crosslinking improved gelling ability and mechanical properties of fish gelatin/GA gels. ${ }^{[15]}$ In our previous study, tannic acid showed strong interactions with EA (mainly hydrogen bonding), indicating a potential to be used as cross-linker for EA/GA stabilized emulsions. ${ }^{[16]}$

Because of the lipophilic nature, astaxanthin is usually dissolved in organic solvents or commercial oils first to prepare the emulsions. ${ }^{[4,9]}$ However, in our study, astaxanthin was naturally dissolved in camelina oil extract, so there is no need for additional oil and toxic organic solvents. To our knowledge, there is no report using EA/GA complex to encapsulate camelina oil extract containing astaxanthin and utilizing tannic acid as the cross-linker to further stabilize the emulsion. Therefore, the objectives of this study were to fabricate EA/GA stabilized emulsion to encapsulate astaxanthin enriched camelina oil extract, characterize the

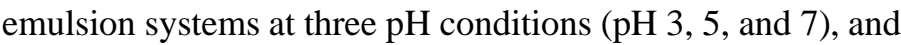
evaluate the physicochemical properties of emulsions. The effect of crosslinking by tannic acid structure and antioxidant functionality of EA/GA stabilized emulsion was also investigated.

\section{Material and methods}

\subsection{Materials}

Camelina oil extract was previously extracted from astaxanthin-enriched camelina seeds provided by the Plant Innovation Center at the University of Nebraska-Lincoln. ${ }^{[5]}$ EA, GA, tannic acid powders, and astaxanthin from Haematococcus pluvialis ( $\geq 97 \%$ purity) were purchased from Sigma-Aldrich Inc. (St. Louis, MO, USA). All other reagents and solvents were of the analytical grade.

\subsection{Emulsion preparation}

EA and GA stock solutions at $1 \%(\mathrm{w} / \mathrm{v})$ and tannic acid stock solution at $10 \%(\mathrm{w} / \mathrm{v})$ were obtained by dissolving powders in deionized water respectively, and mechanically stirred at room temperature for $8 \mathrm{hrs}$. Mixture of EA and GA $(0.5 \% \mathrm{w} / \mathrm{v})$ was achieved by mixing equivalent volume of GA and EA stock solutions, and adjusted to $\mathrm{pH} 7$ using $0.5 \mathrm{~N} \mathrm{NaOH}$. A preliminary oil-in-water emulsion was prepared by homogenizing $1 \%(\mathrm{w} / \mathrm{v})$ camelina oil extract with EA/GA mixture using an Ultra-Turrax T25 high-speed homogenizer (IKA Works, Inc., Wilmington, NC, USA) at 10, $000 \mathrm{rpm}$ for $2 \mathrm{~min}$. The fine emulsions were then obtained after ultrasonic treatment using the ultrasonic homogenizer (Thermofisher, Waltham, MA, USA) at $20 \mathrm{kHz}$ for $2 \mathrm{~min}$ in an ice bath. The whole emulsion preparation process was conducted in the dark to minimize oil degradation by light. The $\mathrm{pH}$ of the emulsion was adjusted to $\mathrm{pH} \mathrm{3,5}$ and 7 with $1 \mathrm{~N} \mathrm{HCl}$ or $0.5 \mathrm{~N} \mathrm{NaOH}$. All final emulsions had a total biopolymer concentration of $1 \%$ $(\mathrm{w} / \mathrm{v})$ and oil content of $1 \%(\mathrm{w} / \mathrm{v})$. For cross-linking, an appropriate volume of tannic acid stock solution was added to the emulsion at $\mathrm{pH} 5$ to achieve a final concentration of 1,2 , and $3 \%(\mathrm{w} / \mathrm{v})$, respectively. Then the $\mathrm{pH}$ of the emulsion was adjusted back to 5 using $0.5 \mathrm{~N} \mathrm{NaOH}$. All emulsions were stored at room temperature in the dark until further analysis.

\subsection{Droplet size and zeta-potential measurements}

The droplet size and zeta-potential of the emulsions were measured by dynamic light scattering (Nano Zetasizer, Malvern Instruments, UK) at $25{ }^{\circ} \mathrm{C}$. To avoid multiple scattering, samples were diluted 10 times using the citrate buffer solution $(0.1 \mathrm{M})$ with the corresponding $\mathrm{pH}$. Samples were vortexed for 30 s and settled for 30 s before measurement. Reported mean droplet diameter and zeta-potential were the average of three independent measurements.

\subsection{Quantification of astaxanthin}

Quantification of astaxanthin content in EA/GA stabilized emulsion was performed according to ${ }^{[9]}$ with minor modifications. To extract astaxanthin, $1.0 \mathrm{~mL}$ of emulsion samples was mixed with $4.0 \mathrm{~mL}$ of organic solvent (dichloromethane: methanol $=2: 1(\mathrm{v} / \mathrm{v})$ ), and then the mixtures were centrifuged at $4000 \mathrm{~g}$ for $20 \mathrm{~min}$ at $25^{\circ} \mathrm{C}$. Astaxanthin was quantified using an Evolution $201 \mathrm{UV}$ Visible Spectrophotometer (Thermo Fisher, Waltham, MA, USA) at $474 \mathrm{~nm}$. The pure dichloromethane and methanol solution $(2: 1, \mathrm{v} / \mathrm{v})$ was used as a blank. The measurements were performed in duplicate. The standard curve of astaxanthin was obtained by dissolving astaxanthin standard in pure dichloromethane and methanol solution $(2: 1, \mathrm{v} / \mathrm{v})$ $\left(\mathrm{R}^{2}=0.992\right)$. Then the entrapment efficiency of astaxanthin in emulsions could be calculated from the standard curve.

The astaxanthin retention was calculated according to Eq. (1):

$$
\text { Astaxanthin retention }(\%)=\frac{C_{t}}{C_{0}} \times 100
$$

where $C_{t}$ is the concentration of astaxanthin in the emulsion at a specific time, while $C_{0}$ is the initial concentration of astaxanthin in oil extract added into emulsions. The measurements were conducted in triplicate.

\subsection{Confocal fluorescence microscopy}

The microstructure of the emulsions was examined using a 
confocal fluorescence microscope (Olympus FV500, Olympus Corporation, PA, USA). Twenty $\mu \mathrm{L}$ of the emulsion was stained with $4 \mu \mathrm{L}$ Nile red and Fast green solution at 0.05 $\mathrm{mg} / \mathrm{mL}$, respectively to dye oil or protein. Then, the mixture was vortexed for $10 \mathrm{~s}$, and stained for $15 \mathrm{~min}$ before image collection. An aliquot $(10 \mu \mathrm{L})$ of the stained emulsion was placed on a microscope slide, covered by a coverslip. Nile red and Fast green were excited at $543 \mathrm{~nm}$ and $488 \mathrm{~nm}$, respectively.

\subsection{Astaxanthin retention against $U V$ light}

To evaluate the stability of the emulsified astaxanthin against UV light, $3 \mathrm{~mL}$ of oil control and emulsion samples in $5 \mathrm{~mL}$ glass vials were placed in a chamber illuminated by a white/UV transilluminator (UVP, LCC, Upland, CA). Astaxanthin retention was measured at an interval of $30 \mathrm{~min}$ as described in section 2.4. Astaxanthin-enriched oil extract dissolved in ethanol was used as the control.

\subsection{Measurement of antioxidant activity}

$\mathrm{ABTS}^{+}$radical cation scavenging activity change of the emulsion due to the heating was measured according to ${ }^{[17]}$. Briefly, potassium persulfate was dissolved at an overall concentration of $2.45 \mathrm{mM}$ in the aqueous solution of $7 \mathrm{mM}$ ABTS. The mixture $\left(\mathrm{ABTS}^{+}\right)$was allowed to stand in the dark $\left(25^{\circ} \mathrm{C}\right)$ for at least $12 \mathrm{~h}$. Then, the $\mathrm{ABTS}^{+}$solution was diluted with PBS buffer to achieve an absorbance of 0.700 ( \pm $0.02) \mathrm{cm}^{-1}$ at $734 \mathrm{~nm}$ and equilibrated at ambient temperature for $30 \mathrm{~min}$. Two $\mathrm{mL}$ of emulsions before and after heat treatment $\left(80{ }^{\circ} \mathrm{C}\right.$ for $\left.5 \mathrm{~min}\right)$ was added to $1 \mathrm{~mL}$ of $\mathrm{ABTS}^{+}$solution and incubated in the dark at room temperature for $6 \mathrm{~min}$. The absorbance was then recorded at $734 \mathrm{~nm}$ using an Evolution 201 UV-Visible Spectrophotometer (ThermoFisher, Waltham, MA, USA). Each measurement was conducted in triplicate. The scavenging of free radical was calculated according to Eq. (2):

Scavenging activity (\%)

$=\frac{\text { Absorbance of control }- \text { Absorbance of sample }}{\text { Absorbance of control }}$

\subsection{Flow behavior of emulsions}

The rheological behavior of emulsion was measured using an MCR 301 rheometer (Anton Parr, Graz, Austria) equipped with a $27 \mathrm{~mm}$ inner diameter concentric cylinder (CC27) at $25{ }^{\circ} \mathrm{C}$. For the oscillatory shear measurements, the linear viscoelastic range of the emulsions was determined by a strain sweep (0.01-20\%) at a constant frequency of $6.28 \mathrm{rad} / \mathrm{s}$. Then, the dynamical oscillatory frequency sweep tests were performed. The frequency (f) was set from 0 to $125 \mathrm{rad} / \mathrm{s}$, and the constant strain amplitude was fixed at $1 \%$ of the linear viscoelastic range. The storage modulus ( $\left.\mathrm{G}^{\prime}\right)$, loss modulus $\left(G^{\prime}\right)$, and loss factor (G'/G') were measured in duplicate.

\subsection{Statistical analysis}

All the measurements were performed in duplicate or triplicate. SAS software (Version 9.4, SAS Institute, Cary, NC, USA) was used for the statistical analysis. The results were subjected to one-way analysis of variance (ANOVA), and the significance level was set at $p<0.05$ for all analyses.

\section{Results and discussions}

\subsection{Characterization of EA/GA stabilized emulsions}

The freshly prepared emulsions showed milky appearance and an orange color due to the presence of astaxanthin. Table 1 summarized the mean hydrodynamic diameter, zeta-potential, and entrapment efficiency of emulsions at different $\mathrm{pH}$. EA/GA stabilized emulsions showed the biggest droplet size

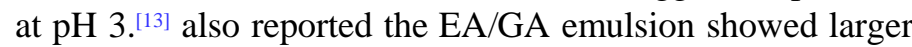
mean hydrodynamic diameter at lower $\mathrm{pH}$. EA and GA could form the insoluble complexes, and then the EA/GA insoluble complexes assembled into larger particles and adsorbed onto the surface of the oil droplets. Besides, emulsions showed the lowest magnitude of zeta-potential $(12.7 \mathrm{mV})$ at this $\mathrm{pH}$, indicating that the flocculation might occur. The emulsion at pH 7 showed smaller diameter and larger magnitude of zetapotential than other two $\mathrm{pH}$ conditions. At $\mathrm{pH} 7$, both EA and GA carried significant negative charges. The magnitude of zeta-potential was over $30 \mathrm{mV}$, showing that it could stabilize the emulsion by strong electrostatic repulsion. ${ }^{[18]}$ In this case, the aggregation of oil droplets was prevented by the repulsive electrostatic interaction resulting in the smallest droplet diameter. ${ }^{[13]}$ reported that EA/GA stabilized sunflower oil emulsions showed the same mean droplet diameter at $\mathrm{pH} 5$ and 7 . The different results obtained in our study might be due to the different oil material and homogenization methods. The entrapment efficiency of astaxanthin in the initial emulsions at three $\mathrm{pH}$ conditions was ranged from 71 to $75 \%$ (Table 1 ). The loss of about $30 \%$ astaxanthin during emulsion preparation may have been a result of oxidation happened during twosteps of homogenization. Some free radicals might be generated during the homogenization resulting in $20 \%$ loss of astaxanthin. ${ }^{[4]}$ The different homogenization methods used in different studies could influence the astaxanthin entrapment efficiency results. The $\mathrm{pH}$ environment did not have a significant impact on astaxanthin in the freshly prepared emulsion.

Table 1. Mean hydrodynamic diameter, zeta-potential, and astaxanthin entrapment efficiency of EA/GA stabilized emulsion.

\begin{tabular}{cccc}
\hline $\mathbf{p H}$ & $\begin{array}{c}\text { Diameter } \\
(\boldsymbol{\mu \mathbf { m } )}\end{array}$ & $\begin{array}{c}\text { Zeta-potential } \\
(\mathbf{m V})\end{array}$ & $\begin{array}{c}\text { Entrapment } \\
\text { Efficiency } \\
(\mathbf{\%})\end{array}$ \\
\hline $\mathbf{3}$ & $5.4 \pm 0.2^{\mathrm{c}}$ & $-12.7 \pm 1.9^{\mathrm{a}}$ & $71 \pm 1^{\mathrm{a}}$ \\
\hline $\mathbf{5}$ & $1.0 \pm 0.0^{\mathrm{b}}$ & $-17.0 \pm 1.7^{\mathrm{b}}$ & $72 \pm 2^{\mathrm{a}}$ \\
\hline $\mathbf{7}$ & $0.2 \pm 0.0^{\mathrm{a}}$ & $-37.2 \pm 1.5^{\mathrm{c}}$ & $75 \pm 2^{\mathrm{a}}$ \\
\hline
\end{tabular}

Values followed by the different letters indicate significant difference $(\mathrm{p}<0.05)$.

Confocal fluorescence microscopy is a practical tool to observe the microstructure of the emulsions, therefore to 
investigate the interaction between oil and protein in the formulation. The images obtained by confocal fluorescence microscopy were shown in Fig. 1. In all images, the oil droplets were shown in red and proteins were indicated in green. The inter-fat droplet distribution mainly depended on the $\mathrm{pH}$ condition. At $\mathrm{pH} 3$ and 5, protein aggregation seemed to be the dominating phenomenon. However, at $\mathrm{pH} 7$, the oil droplets were mainly coated by individual proteins rather than protein aggregates. The results supported the droplet size observation that the strong electrostatic repulsion prevented the protein aggregation at $\mathrm{pH} 7$.

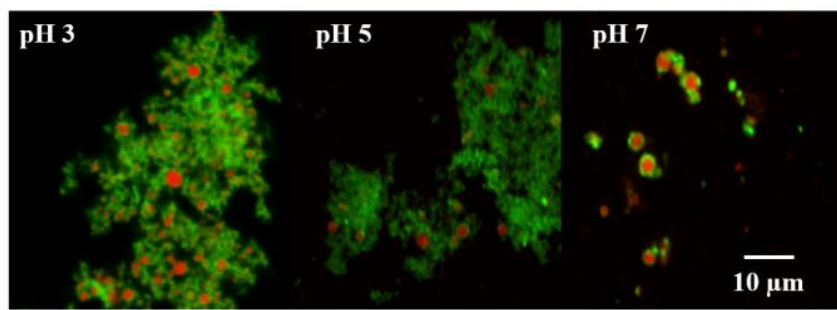

Fig. 1 Confocal fluorescence microscopy images of EA/GA stabilized emulsions at three $\mathrm{pH}$ conditions. Lipid in red and protein in green.

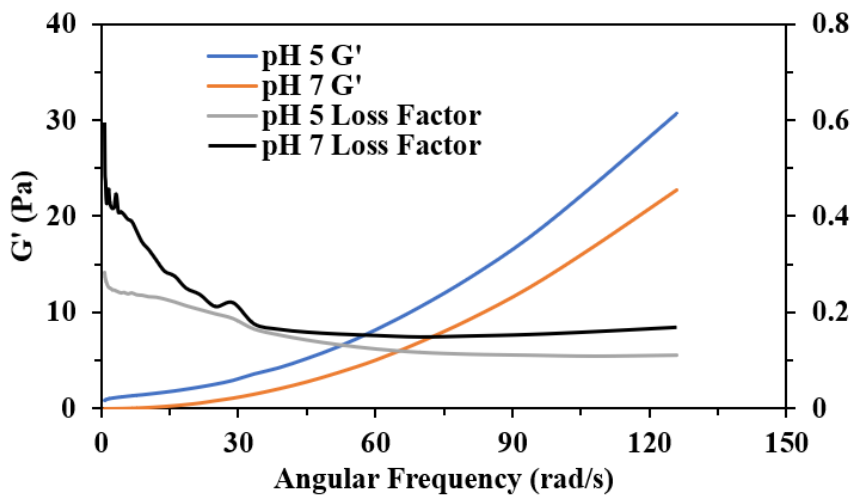

Fig. 2 Storage modulus (G') and loss factor curve of EA/GA stabilized emulsions at $\mathrm{pH} 5$ and 7.

The flow behavior of EA/GA stabilized emulsion at $\mathrm{pH} 5$ and 7 was also studied (Fig. 2). The flow behavior test of the emulsion at $\mathrm{pH} 3$ was not recorded because of precipitation occurring during the test. The flow behavior is one of the most important properties of emulsions. ${ }^{[19]}$ Appearance, texture and shelf life of emulsion products are often related to their rheological properties. Small amplitude oscillatory shear tests were performed to study the rheological properties of emulsions. ${ }^{[19,20]}$ The storage modulus $G^{\prime}$ is a measure of the energy stored reversibly within in the system, characterizing the elastic behavior, while the loss modulus G" represents the viscous behavior. The loss factor $\left(G^{\prime \prime} / G^{\prime}\right)$ describes the relationship between the viscous and elastic portion of the sample. As shown in Fig. 2, in both cases, the G' were higher than G", and loss factor values were less than 1 , representing a more elastic or gel-like system. The emulsion at $\mathrm{pH} 5$

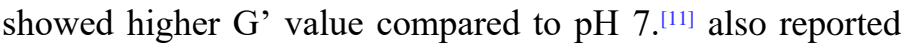
that EA/GA stabilized emulsion possessed a higher $G$ ' value at $\mathrm{pH} 4.8$ than $\mathrm{pH} 7$ because the emulsion droplets might have better rigidity at $\mathrm{pH} 4.8$. The loss factor of the emulsion at $\mathrm{pH}$ 5 remained relatively stable within the range of angular frequency, which might reflect a better emulsion stability. ${ }^{[11]}$ From the frequency study, the stability of emulsions may follow the order: $\mathrm{pH} 5>\mathrm{pH} 7>\mathrm{pH} 3$.

\subsection{Droplet size, zeta-potential change, and astaxanthin retention during storage}

Fig. 3 shows the change of droplet size (a), zeta-potential (b), and astaxanthin retention (c) of EA/GA stabilized emulsions during storage for 6 days at room temperature at three $\mathrm{pH}$ conditions. As shown in Fig. 3 (a), at pH 3, the mean droplet diameter slightly decreased as the storage time increased, which may due to the precipitation of emulsions. But the mean droplet size was still above $4 \mu \mathrm{m}$. At $\mathrm{pH} 5$, the mean droplet diameter and zeta-potential remained stable during storage. When $\mathrm{pH}$ changed to 7 , the droplet size increased due to the increased zeta-potential during storage. The increased zetapotential of droplets during storage might be associated with the degradation of astaxanthin.

At $\mathrm{pH} 3$, due to the large droplet size, gravity-induced particle precipitation might be the main factor resulting in the decrease of astaxanthin retention. Although the insoluble EA/GA complexes absorbed onto the surface of the oil droplets, they were not stable enough to form the Pickering emulsion in this case. At pH 5 and 7, EA and GA carried similar net charges and no electrostatic attractive interactions occurred, resulting in a thin and loose interfacial layer. ${ }^{[13]}$ However, after 6 days' storage, emulsion at pH 5 showed the highest astaxanthin retention. The phenomenon might be explained by relative stable droplet size, zeta-potential, and flow behavior compared to the emulsion at $\mathrm{pH} 7$.

Another point was that the astaxanthin retention was not only affected by the emulsion stability, but also the degradation of astaxanthin. Our observation confirmed that the degradation of astaxanthin might still occur during the storage even though the emulsions were stored in the dark. In addition, ${ }^{[21]}$ reported that the degradation of astaxanthin might be associated with lower oxidative stability of fatty acids in the oil. If lipid oxidation could take place due to the high omega-3 oil content of camelina oil, the formation of highly reactive compounds, like peroxyl radicals, could increase the degradation of astaxanthin. ${ }^{[22]}$ observed that the oxidation of vegetable oil increased the degradation of lutein. Limiting the oxidation of camelina oil and decreasing astaxanthin degradation could be achieved by flushing the astaxanthin emulsion with nitrogen gas resulting in an oxygen-free atmosphere. However, exclusion of oxygen from food beverage during processing and storage is often not practical, and once the bottle is open, it would become exposed to oxygen. ${ }^{[23]}$

\subsection{Effect of crosslinking by tannic acid on EA/GA stabilized emulsion at pH 5}



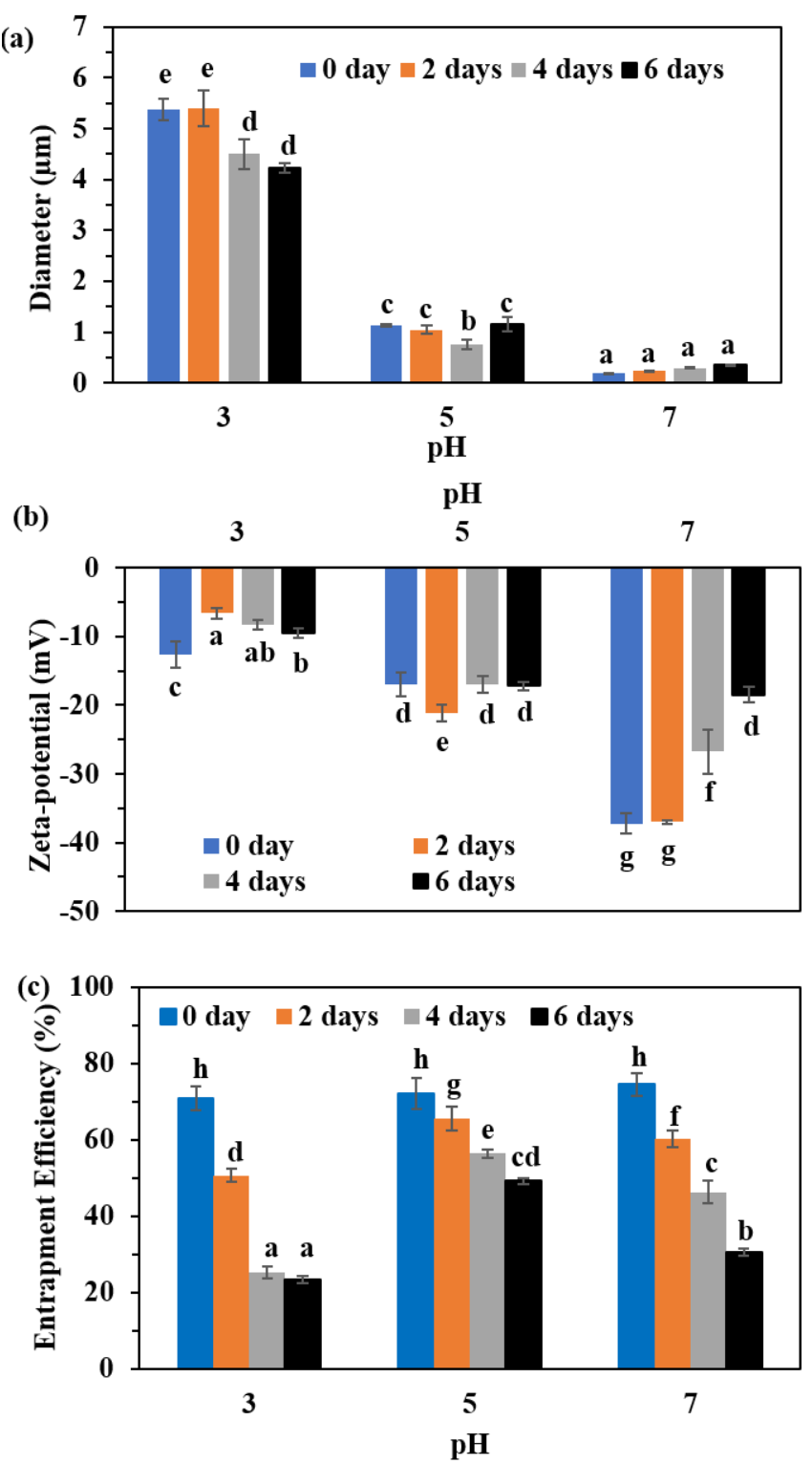

Fig. 3 Mean droplet diameter (a), zeta-potential (b), and astaxanthin retention (c) change of EA/GA stabilized emulsions during room temperature storage. Different lowercase letters mean significant differences $(p<0.05)$.

\subsubsection{Droplet size and entrapment efficiency}

EA/GA stabilized emulsion at $\mathrm{pH} 5$ was further crosslinked by tannic acid. The effect of crosslinking on droplet size and entrapment efficiency change was studied, and the results are shown in Fig. 4. After the crosslinking, tannic acid formed linkages with EA/GA complexes. Only 2\% and 3\% tannic acid increased the mean droplet diameter of the emulsions significantly. Perhaps $1 \%$ of tannic acid was not sufficient to crosslink EA/GA and form complexes in the emulsion system. An appropriate tannic acid: biopolymer ratio should also be considered for the utilization of tannic acid as cross-linker. The addition of tannic acid did not affect the entrapment efficiency, further confirmed our hypothesis that loss of astaxanthin mainly happened during emulsification. As a cross-linker, tannic acid did not interact with astaxanthin directly because the oil droplets were surrounded by the interfacial layer consisting of EA/GA complexes. In general, tannic acid can interact with proteins through non-covalent interactions (such as hydrogen bonding, electrostatic interaction, and hydrophobic interaction). ${ }^{[16]}$ Based on the previous study, hydrogen bonds might be the main bonding force between EA and tannic acid, ${ }^{[16]}$ which might be one of the major forces to interact with EA/GA complexes as shown in Fig. $5{ }^{[15]}$ reported that the hydroxyl groups or aromatic rings of tannic acid might be more reactive toward protein molecules into the fish gelatin-GA complex coacervates. However, further experiments will be needed to determine the binding mechanism between tannic acid and EA/GA complex.

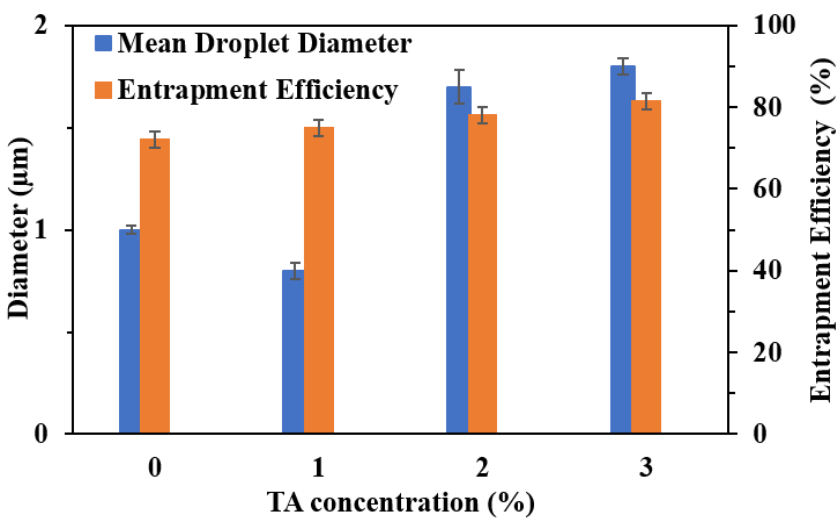

Fig. 4 Effect of tannic acid on droplet size and entrapment efficiency of EA/GA stabilized emulsion at $\mathrm{pH} 5$. Different uppercase letters mean significant differences $(p<0.05)$ of the mean droplet diameter. Different lowercase letters mean significant differences $(p<0.05)$ of the entrapment efficiency.

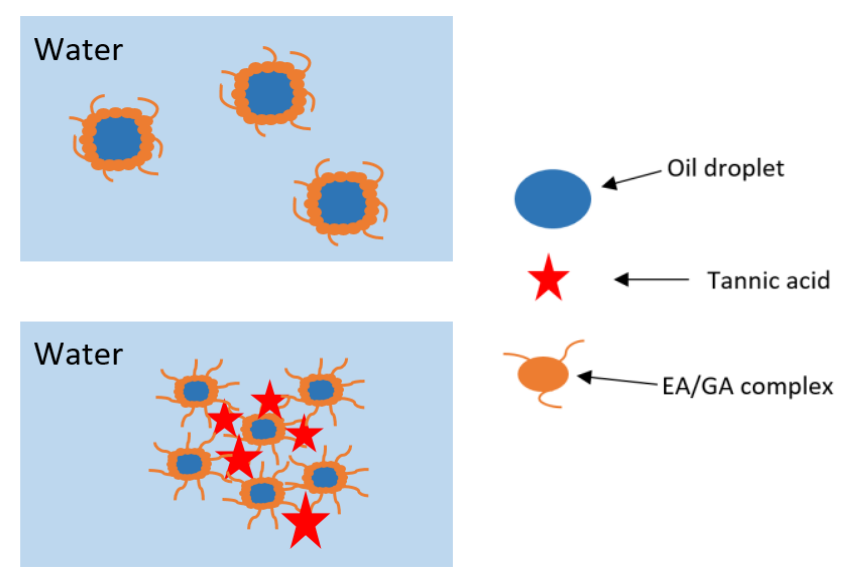

Fig. 5 A proposed mechanism diagram of EA/GA stabilized emulsion with and without tannic acid crosslinking.

\subsubsection{Protection of astaxanthin against UV light}

Several mechanisms can result in the degradation of carotenoids in different systems when exposed to UV light. One possible mechanism is that the light may produce carotenoid radicals because of hydrogen abstraction, which caused the bleaching of carotenoids. ${ }^{[4,23]}$ The retention of astaxanthin in emulsions against UV light exposure is shown in Fig. 6. Regardless of the effect of tannic acid, in all three 
$\mathrm{pH}$ conditions, the emulsion systems helped astaxanthin against photodegradation.

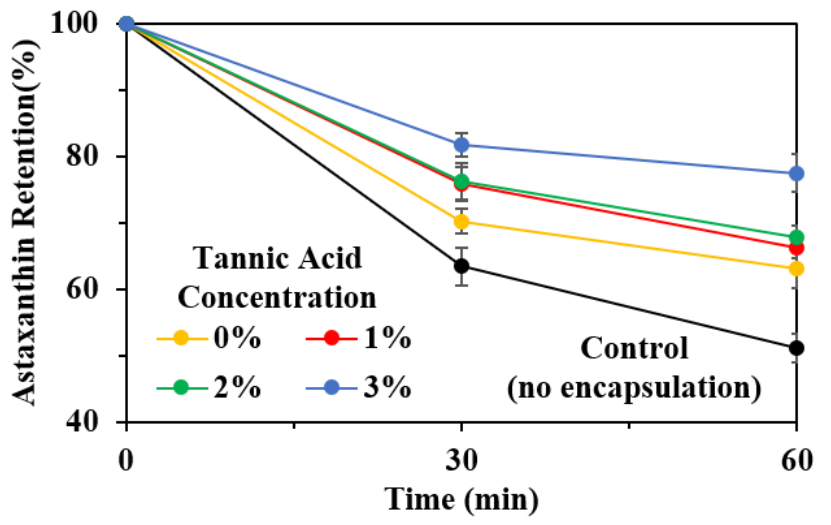

Fig. 6 Effect of tannic acid on astaxanthin retention against UV light of EA/GA stabilized emulsion at $\mathrm{pH} 5$.

As shown in Fig. 6, astaxanthin retention after UV light exposure was improved by tannic acid crosslinking, and the 3\% tannic acid gave the best protection of astaxanthin against UV light. As the tannic acid concentration increased, the emulsion formed a more compact network, which could reduce light penetration. In addition, in the presence of singlet oxygen generators, light can degrade carotenoids very effectively. ${ }^{[24]}$ Phenolic compounds are also oxidized by singlet oxygen. In this case, the oxidation of tannic acid could also occur in this case. Once the tannic acid oxidized, the color of emulsion became intensive which could limit the light penetration. However, if there were not any singlet oxygen generators in the system, the decomposition of carotenoid through this pathway could be neglected. ${ }^{[23]}$

\subsubsection{Protection of astaxanthin against heat stress}

Thermal processing is very common for commercial food and beverage emulsions. The thermal treatment may increase the degradation of astaxanthin and camelina oil resulting in low antioxidant activity. In addition, the denaturation of EA happens at around $80^{\circ} \mathrm{C}$, which may also affect the encapsulation system. Thus, it is very important to study the influence of heat on the antioxidant activity of EA/GAstabilized emulsions and emulsion stabilized by EA alone. The effect of tannic acid cross-linking was also examined in this study. As shown in Fig. 7 (a), before heating, the EAstabilized emulsion had the lowest antioxidant activity, because the emulsion was not stable at $\mathrm{pH}$ 5. Other treatments did not show any significant difference in antioxidant activity before heating. After the thermal processing, the EAstabilized emulsion still had the lowest antioxidant activity $(\sim 17.8 \%)$, and it was even worse than the control (no encapsulation). The pI of EA was around 5, so the protein precipitation was more likely to occur resulting in unstable emulsion and less antioxidant activity. However, the incorporation of GA improved the emulsion stability at $\mathrm{pH} 5$. In the EA/GA stabilized emulsions, EA/GA complexes formed the interfacial layer, which overcame the limitation of using EA at $\mathrm{pH}$ 5. The EA/GA emulsion system was more effective than control and EA to protect astaxanthin against heat stress. In addition, as the concentration of tannic acid in the emulsion increased, the antioxidant activity also increased. Tannic acid belongs to the polyphenol group and acts as an antioxidant through single electron transfer. ${ }^{[25]}$ ABTS method detects antioxidant activity from both carotenoids and polyphenols. In this case, astaxanthin and tannic acid both contributed to the antioxidant activity of the system.
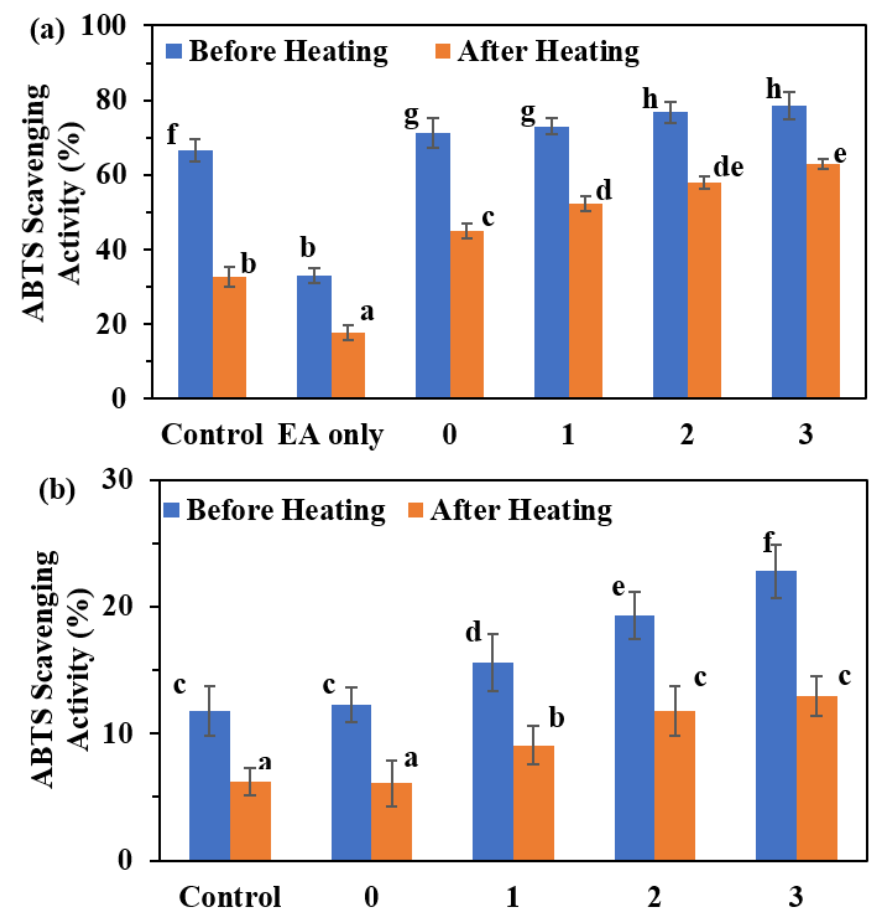

Fig. 7 Effect of tannic acid on ABTS scavenging antioxidant activity against the heat of EA/GA stabilized emulsion at $\mathrm{pH} 5$. (a) astaxanthin-enriched camelina oil; (b) non-engineered camelina oil (no astaxanthin). Control: no encapsulation. EA only: EA stabilized emulsion. Sample numbered 0, 1, 2, 3 refer to EA/GA stabilized emulsion with $0 \%, 1 \%, 2 \%$ and $3 \%$ tannic acid, respectively. Different lowercase letters mean significant differences $(\mathrm{p}<0.05)$ of the ABTS scavenging activity.

Tocopherols are important minor lipid components in camelina seed, and best known for their antioxidant activities. ${ }^{[5]}$ Non-engineered camelina seed oil was also used in this study in order to examine the contribution to the antioxidant activity from tocopherols. Compare Fig. 7 (a) with (b), either with or without encapsulation, regular camelina oil contained much lower antioxidant activity $(\mathrm{P}<0.05)$, which agreed with the results from. ${ }^{[5]}$ After the crosslinking by tannic acid, the antioxidant activity of emulsions was improved.

\subsubsection{Flow behavior}

The effect of tannic acid on emulsion flow behavior was studied by frequency sweeping test. As shown in Fig. 8, the loss factor $\left(G^{\prime \prime} / G^{\prime}\right)$ of all the emulsions was less than 1 , indicating a more elastic behavior than viscous behavior. But the loss factor value was greater than 0.1 , showing a typical week gel behavior of dressings and emulsions. ${ }^{[26]}$ After crosslinking by $3 \%$ tannic acid, emulsion at $\mathrm{pH} 5$ showed 
different flow behavior compared to other emulsions. As the frequency increased, the loss factor of emulsion with $3 \%$ tannic acid at $\mathrm{pH} 5$ was also increased, whereas the loss factor of other emulsions decreased. Although the elastic gel-like behavior was still dominant in all emulsions, the viscous portion increased in the emulsion with $3 \%$ tannic acid at $\mathrm{pH} 5$. Generally, the crosslinking by tannic acid increased the loss factor at each $\mathrm{pH}$ condition, which indicated that the network structure became easier to rearrange to accommodate the strain after crosslinking. ${ }^{[27]}$ Because of the weak gel structure, macromolecules interconnections and entanglements could be disrupted by applying high shear rates. ${ }^{[26]}$ Our rheological analysis results partially agreed with the results from others. ${ }^{[11]}$

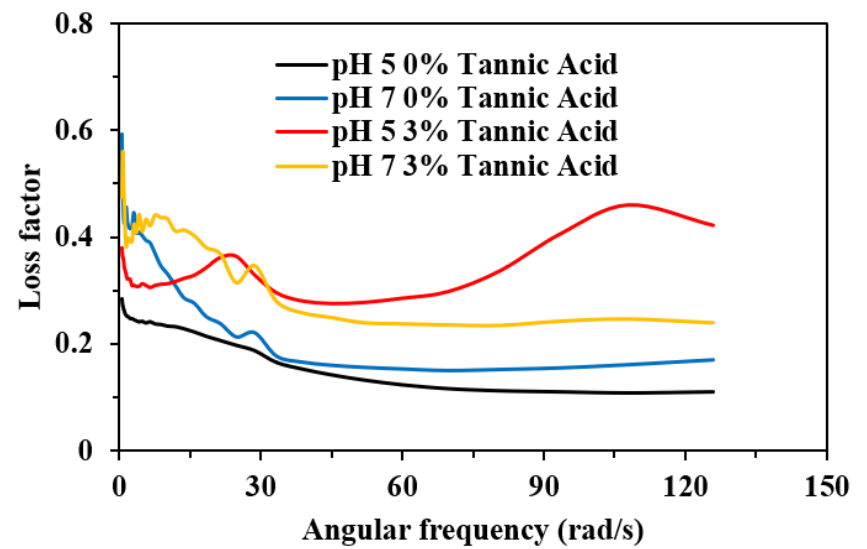

Fig. 8 Flow behavior of EA/GA stabilized emulsions at pH 5 and 7 with and without crosslinking by $3 \%$ tannic acid.

\section{Conclusions}

Astaxanthin-enriched camelina oil extract, as a potential astaxanthin source, was emulsified using a mixture of EA/GA with and without tannic acid cross-linking. The emulsion showed the smallest droplet size at $\mathrm{pH} 7$, but astaxanthin showed the highest retention at $\mathrm{pH} 5$ during storage. EA/GA stabilized emulsion was able to protect astaxanthin against UV light effectively and reduce the deduction of antioxidant activity after heating. The crosslinking by tannic acid further improved the protection of astaxanthin and increased the antioxidant activity. But it also changed the droplet size and flow behavior of the emulsion. The resultant emulsion product may have the potential to be used as a food ingredient in food applications.

\section{References}

[1] Q. Shen and S. Y. Quek, J. Food Eng., 2014, 123, 165-171, doi: https://doi.org /10.1016/j.jfoodeng.2013. 09.002.

[2] N. Khalid, G. Shu, I. Kobayashi, M. Nakajima and C. J. Barrow, Colloids and Surfaces B: Biointerfaces, 2017, 157, 355-365, doi: https://doi.org/10.1016/j. colsurfb.2017.06.003.

[3] C. Bustos-Garza, J. Yáñez-Fernández and B. E. Barragán-Huerta, Food Res. Inter, 2013, 54, 641-649,doi: https://doi.org/10.1016/j.food res.2013.07.061.
[4] X. Liu, D. J. McClements, Y. Cao and H. Xiao, Food Biophys., 2016, 11, 302-310, doi: 10.1007/s11483- 016-9443-6.

[5] L. Xie, E. Cahoon, Y. Zhang and O. N. Ciftci, J. Supercritical Fluids, 2019, 143, 171-178, doi: https://doi.org/10.1016/j.supflu.2018. 08.013.

[6] H. Belayneh, R. Wehling, E. Cahoon and O. Ciftci, J. Supercritical Fluids, 2015, 104, 153-159, doi: https://doi.org/10.1016/j.supflu.2015.06.002.

[7] M. Li, M. R. Zahi, Q. Yuan, F. Tian and H. Liang, Eur. J. Lipid Sci. Technol., 2016, 118, 592-602, doi: 10.1002/ejlt.201400650.

[8] C. Kamezaki, A. Nakashima, A. Yamada, S. Uenishi, H. Ishibashi, N. Shibuya, S. Hama, S. Hosoi, E. Yamashita and K. Kogure, J. Clin. Biochem. Nutr., 2016, 59, 100-106, doi: 10.3164/jcbn.15153.

[9] N. Khalid, G. Shu, B. J. Holland, I. Kobayashi, M. Nakajima and C. J. Barrow, Food Res. Inter., 2017, doi: https://doi.org/10.1016/j. foodres.2017.06.019.

[10] F. Liu, C. Ma, R. Zhang, Y. Gao and D. Julian McClements, Food Chem., 2017, 221, 395-403, doi: https://doi.org/ 10.1016/j.foodchem.2016.10.057.

[11] F. Niu, D. Niu, H. Zhang, C. Chang, L. Gu, Y. Su and Y. Yang, Food Hydrocolloids, 2016, 52, 607-614, doi: https://doi.org/10.1016/j.foodhyd.2015.08.010.

[12] B. H. Ali, A. Ziada and G. Blunden, Food Chem. Toxicol., 2009, 47, 1-8, doi: https://doi.org/10.1016/j. fct.2008.07.001.

[13] F. Niu, Y. Zhang, C. Chang, W. Pan, W. Sun, Y. Su and Y. Yang, Food Hydrocolloids, 2017, 63, 602-610, doi: https://doi.org/10.1016/j.foodhyd.2016.10.007.

[14] B. Zeeb, M. Gibis, L. Fischer and J. Weiss, Food Hydrocolloids, 2012, 27, 126-136, doi: https:// doi.org/10.1016/j.foodhyd.2011.08.005.

[15] M. Anvari and D. Chung, Food Hydrocolloids, 2016, 60, 516-524, doi: https://doi.org/10.1016/j.food hyd.2016.04.028.

[16] L. Xie, R. L. Wehling, O. Ciftci and Y. Zhang, Food Res. Inter, 2017, 102, 195-202, doi: https://doi.org/10. 1016/j.foodres.2017.10.007.

[17] L. Wang, P. Gulati, D. Santra, D. Rose and Y. Zhang, Food Chem., 2018, 240, 1039-1046, doi: https://doi.org/10.1016 /j.foodchem.2017.08.036.

[18] L. Wang and Y. Zhang, J. Agric. Food Chem., 2017, 65, 2990-2998, doi: 10.1021/acs.jafc.7b00194.

[19] J. Feng, H. Cai, H. Wang, C. Li and S. Liu, Food Chem., 2018, 241, 60-69, doi: https://doi.org/10. 1016/j.foodchem. 2017.08.055.

[20] F. Liu, D. Wang, C. Sun and Y. Gao, Food Hydrocolloids, 2016, 52, 661-669, doi: https://doi. org/10.1016/j.foodhyd.2015. 08.007.

[21] S. Takeungwongtrakul and S. Benjakul, Inter. Aquatic Res., 2016, 8, 15-27, doi: 10.1007/s40071-015-0120-z.

[22] R. Lavecchia and A. Zuorro, Chem. Eng. Trans., 2008, 14, e204.

[23] N. Anarjan and C. P. Tan, J. Am. Oil Chem. Soc., 2013, 90, 12231227, doi: 10.1007/s11746-013-2270-8.

[24] E. Choe and D. B. Min, Comprehensive Rev. Food Sci. Food Safety, 2009, 8, 345-358, doi: 10.1111/j.1541-4337.2009.00085.x.

[25] K. Goiris, K. Muylaert, I. Fraeye, I. Foubert, J. De Brabanter and L. De Cooman, J. Appl. Phycology, 2012, 24, 1477-1486, doi: 10.1007/ s10811-012-9804-6. 
[26] I. G. Mandala, T. P. Savvas and A. E. Kostaropoulos, J. Food Eng., 2004, 64, 335-342, doi: https://doi.org/10.1016/j.jfoodeng.2003.10.018.

[27] G. Bortnowska, J. Balejko, G. Tokarczyk, A. Romanowska-Osuch and N. Krzemińska, Food Hydrocolloids, 2014, 36, 229-237, doi: https:// doi.org/10.1016/j.foodhyd.2013.09.012.

\section{Author information}

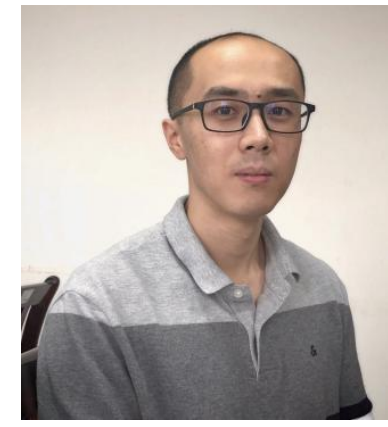

Liyang Xie received his M.S. in Food Science and Technology in 2019 from the University of NebraskaLincoln (United States). He is working at Shaanxi Product Quality Supervision and Inspection Research Institute (China). His previous research interest mainly focused on the encapsulation of astaxanthin in protein-polysaccharide-based emulsions.

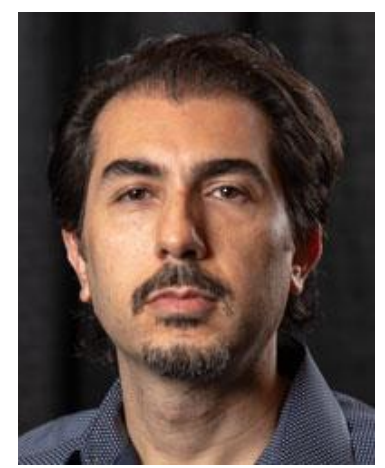

Ozan Ciftci is an Associate Professor in the Department of Food Science and Technology at the University of Nebraska-Lincoln (United States). Dr. Ciftci obtained his B.S., M.S., and Ph.D. from University of Gaziantep, Turkey and finished his postdoc training at the University of Lethbridge, Canada. He joined UNL as a faculty member in 2014. The main goal of his research group is the green processing of lipids to produce novel food products with improved function and efficacy.

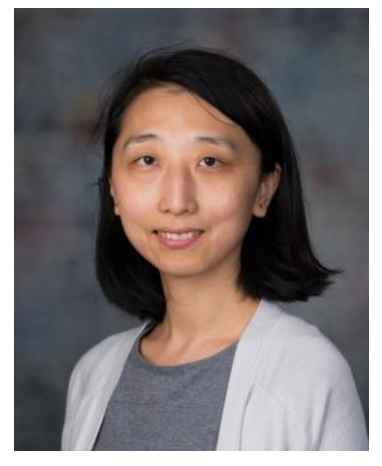

Yue Zhang is a Professor in the School of Food Science and Biotechnology at Zhejiang Gongshang University (China). Dr. Zhang obtained her B.S. in Chemistry and Ph.D. in Physical Chemistry from Wuhan University (China). She worked as a Postdoctoral Research Associate at the University of Tennessee (United States) and Assistant Professor at the University of Nebraska-Lincoln (United States). Dr. Zhang's research mainly focuses on the physicochemical properties of food biopolymers and the development of biopolymer-based nanoformulations for targeted delivery purpose.

Publisher's Note: Engineered Science Publisher remains neutral with regard to jurisdictional claims in published maps and institutional affiliations. 